Check for updates

Cite this: Mater. Adv., 2022, 3, 1629

Received 29th November 2021, Accepted 14th December 2021

DOI: $10.1039 / \mathrm{d} 1 \mathrm{ma01121a}$

rsc.li/materials-advances

\title{
Removal of highly dispersive microspheres from wastewater using modified spirulina hetero- coagulation and their application in flame retardancy $\dagger$
}

\author{
Gaoda Zheng, ${ }^{\text {ab }}$ Zhihao Cai, ${ }^{\mathrm{b}}$ Ziyi Yu, ${ }^{\mathrm{b}}$ Yuqin Yang, ${ }^{\mathrm{b}}$ Hui Ye, ${ }^{\mathrm{b}}$ Zheng Qin, \\ Shiyu Zhang ${ }^{*}{ }^{\mathrm{b}}$ and Xiaobing Zuo*ab
}

\begin{abstract}
There is an urgent demand to find an effective solution to microplastic pollution in water. In addition, the harm of algal flooding to water quality also haunts human society because of the increase of industrialization. In this work, modified spirulina (T-spirulina) is used to destroy the electrostatic doublelayer structure of microspheres by a hetero-coagulation process, completely removing highly dispersed poly(tetrafluoroethylene) (PTFE/PSAN) microspheres from water. Experimental results indicate that the PTFE/PSAN microspheres could be controllably deposited on the surface of T-spirulina with the assistance of formic acid. T-Spirulina absorbs at least three times its own mass of PTFE/PSAN microspheres and the removal efficiency of the highly dispersed microspheres in water is even up to $99.81 \mathrm{wt} \%$, which is better than that of other demulsifiers. Due to the fact that T-spirulina is rich in nitrogen and phosphorus elements, the hetero-coagulation product (STP) has great potential in the field of flame retardants. The thermogravimetric analysis (TGA) tests show that the addition of STP could improve the flame retardant performance of an epoxy resin (EP) matrix. Meanwhile, the limiting oxygen index (LOI) and vertical combustion performance are also improved with an increase of the amount of PTFE/PSAN coating used. With the addition of $5 \mathrm{wt} \%$ of STP, the LOI value of the composite increased up to 22.8. Cone calorimetry shows that $\mathrm{HRR}, \mathrm{CO}$ and $\mathrm{CO}_{2}$ production are significantly decreased with the STP10 composite, which is much lower than that of neat EP.
\end{abstract}

\section{Introduction}

We are living in a polymer age. Since Staudinger came up with the original concept of "macromolecules", polymers have shaped human life everywhere in our daily lives. ${ }^{1}$ Microplastics are recognized as more intractable contaminants, not only because of their long lifetime and low degradability but also because of their interactions with other pollutants in the environment. $^{2-5}$ There have been a series of research papers that have continuously reported about the technical solutions available for microplastics removal. ${ }^{6-10}$ Biodegradable polymers are regarded as the most feasible solution to the damage caused by microplastics. However, many types of biodegradable polymers, such as poly(lactic) acid (PLA) and poly(caprolactone)

\footnotetext{
${ }^{a}$ College of Chemistry, Chemical Engineering and Materials Science, Soochow University, Suzhou 215123, Jiangsu, P. R. China

${ }^{b}$ School of Materials Engineering, Changshu Institute of Technology, Suzhou 215500, P. R. China.E-mail: zhangshiyu@cslg.edu.cn, zuoxiaobing@cslg.edu.cnn $\dagger$ Electronic supplementary information (ESI) available. See DOI: 10.1039/ d1ma01121a
}

(PCL), can degrade only under extremely harsh conditions, and they failed to show any biodegradation in home composting or in the marine environment. ${ }^{10}$ This means that the value of many of the world's best-selling biodegradable plastics needs to be reassessed. Raikova et al. recently came up with a clever solution that aims to co-liquefy macroalgal species with common microplastics, such as poly(ethylene) (PE), poly(propylene) (PP) and poly(amide) (PA), for regeneration of biofuels or monomers. ${ }^{8}$ Nevertheless, the whole liquefaction process is carried out under hydrothermal conditions, because its largescale applications would be in the environmental protection industries.

Microspheres are a type of microplastics that are widely used in chemical engineering, personal care and cosmetic products. ${ }^{11}$ Due to their tiny size, microspheres present in the hydrographic net are hard to remove, and always transfer adsorbed organic pollutants to aquatic species causing ecological damage. ${ }^{12}$ Under existing approaches, chemical enterprises require expensive tertiary treatments capable of cleaning microbeads from effluent streams, but there is a lack of mandatory supervision and infrastructure for their use, 
especially in developing countries. Thus, it is urgent that an inexpensive and energy-efficient solution is sought that can be used both locally and to supplant existing tertiary treatments. Coagulation techniques provide a series of cost-effective solutions for tertiary treatment of microspheres, and rarely depend on advanced equipment or harsh working conditions. ${ }^{13-15}$ Zhou et al. reported systematic research about the removal performance and coagulation mechanism of poly(styrene) (PS) and PE microbeads in natural water. ${ }^{15}$ Polyaluminium chloride (PAC) and ferric chloride $\left(\mathrm{FeCl}_{3}\right)$ when used as coagulants demonstrate differences in the removal efficiency of PS and PE microbeads. Nevertheless, unlike PS and PE, many microspheres have high compatibility in the freshwater systems. Due to the adhesion of surfactants and compatible copolymers, modified fluoropolymer microspheres, indispensable raw materials in the electrical industry, are dispersed well in the emulsion. ${ }^{16}$ There is still a significant challenge for the remediation of stubborn microspheres.

Meanwhile, algal blooms are also environmentally problematical and excessive to dispose with the increased nitrogen and phosphorus concentrations in discharged effluents. ${ }^{17}$ Algae not only represent a vast untapped resource for the production of renewable chemicals and fuels, but they can also be applied for removing arsenates and eutrophication of water bodies. ${ }^{18,19}$ Spirulina is a marine alga, Arthrospira platensis, with a naturally intact three-dimensional helical structure and a number of researchers have demonstrated its feasibility and efficiency for contaminant removal..$^{20-22}$ As an example, Doke et al. showed that by using a spirulina strain they could manage to remove up to $95 \%$ of biochemical oxygen demand, $52 \%$ of nitrate, and $76 \%$ of phosphate, while reducing the bacterial count up to $75 \% .^{23}$ As mentioned previously, due to emulsifiers adhering to fluororesin microspheres, it is not possible for fluororesin microspheres to coagulate completely. With the assistance of the high adhesiveness of spirulina, it is thought that the modified spirulina can weaken the electrostatic double-layer structure of the fluororesin microspheres and simultaneously enhance the interaction between the microbeads in an aqueous emulsion during the hetero-coagulation process, facilitating the controllable aggregation of the microbeads on the spirulina surface. In particular, compared to the fluororesin microspheres with definite charges, spirulina obviously promoted a hetero-coagulation process when modified with anisotropic charges.

The PTFE is often used as an antidripping and synergistic agent in polymer flame retardant formulations, however, due to its low surface energy and poor compatibility with other resins, ${ }^{24,25}$ PTFE is usually coated with a polymer, such as PMMA, PSAN, to improve its compatibility. ${ }^{26-28}$ The PTFE/PSAN anti-drip agent produced by Changshu ZhenFu New Materials has been introduced to the commercial market. At present, after the condensation of the PTFE/PSAN emulsion, the residual rate of PTFE/PSAN emulsion is too high, and filtration membrane treatment is adopted in industry, which leads to a high cost for post-treatment and certain pollution of water resources.
Herein, a smart and green approach for the controllable coagulation of fluororesin microspheres on the spirulina surface with a stable core-shell structure, produced with the help of a hetero-coagulation process, is proposed. The aim of this research is to realize the removal of various wastes in water. Hence, modified fluoropolymer microspheres, such as polytetrafluoroethylene (PTFE) coated with a copolymer of styrene and acrylonitrile (PTFE/PSAN), are gaining excellent dispersivity, and were chosen as target pollutants for waste recycling. Benefiting from the high fluorine, nitrogen and phosphorus contents in the core-shell structure, the hetero-coagulation product exhibits an excellent flame-resistant and antidripping potential as an environmentally-friendly flame retardant. Before the hetero-coagulation was commenced, the spirulina surface was treated with tetradecyl trimethyl ammonium bromide (TTAB) which helped to give a high removal efficiency of the fluororesin microspheres. During the heterocoagulation process, the TTAB adsorbed onto the spirulina surface not only promotes spirulina dispersion but also accelerates the whole hetero-coagulation system as a demulsifier. The modified spirulina absorbs at least three times its own mass of the PTFE/PSAN microspheres by a hetero-coagulation process and, more importantly, the removal efficiency of the highly dispersed microspheres in water can even reach up to $99.81 \mathrm{wt} \%$. This work further demonstrates the feasibility of the hetero-coagulation process for a controllable core-shell structure of the product and its excellent flame-resistant and antidripping properties.

\section{Experimental section}

\section{Materials}

The PTFE emulsion (30 wt\%) was provided by the Shanghai $3 \mathrm{~F}$ New Materials (China). Styrene was purchased from the Shanghai Lingfeng Chemical Reagent Company. Acrylonitrile was supplied by the Chengdu Xiya Reagent Company. Alkyl polyoxyethylene ether (S13), for use as an emulsifier, was purchased from the Shanghai Miura Chemical Company. Formic acid, $\mathrm{H}_{2} \mathrm{SO}_{4}, \mathrm{NaOH}, \mathrm{KCl}, \mathrm{CaCl}_{2}, \mathrm{Al}_{2}\left(\mathrm{SO}_{4}\right)_{3}$, sodium lauryl sulfate (SDS), TTAB, and ammonium persulfate $\left(\left(\mathrm{NH}_{4}\right)_{2} \mathrm{~S}_{2} \mathrm{O}_{8}\right)$, used as an initiator, were supplied by the Sinopharm Group Chemical Reagent Company. Spirulina platensis was obtained from the Nanjing Kangmanlin Chemical Industry Company. The AB glue was purchased from the Dongguan Huirui Rubber Industry Company.

\section{Preparation of sea urchin-like structured PTFE/PSAN emulsion}

The SDS (1.00 g), styrene (16.00 g), acrylonitrile (4.00 g) and deionized water $(40.00 \mathrm{~mL})$ were mixed in a $250 \mathrm{~mL}$ threenecked flask with simple stirring at room temperature for $60 \mathrm{~min}$, making a stable pre-emulsion.

The PTFE emulsion (20.00 g) and the pre-emulsion $(6.10 \mathrm{~g})$ were mixed in a three-necked flask with stirring. Then, the $\left(\mathrm{NH}_{4}\right)_{2} \mathrm{~S}_{2} \mathrm{O}_{8}$ initiator solution $(30.00 \mathrm{~mL})$ which a concentration of $1.00 \mathrm{~g} \mathrm{~L}^{-1}$ was added into the three-necked flask. The 
remaining pre-emulsion (54.90 g) was slowly instilled into the mixed dispersion. The dropwise addition was completed within $2 \mathrm{~h}$ at $70{ }^{\circ} \mathrm{C}$. When the addition was completed, the temperature rose to $75{ }^{\circ} \mathrm{C}$. Then, the remaining initiator was added and the reaction was continued for $2-4 \mathrm{~h}$ to increase the monomer conversion rate. After the reaction finished, the mixed dispersion was filtered and cooled to room temperature. The solid content of the prepared PTFE/PSAN mixed emulsion was about $33.00 \mathrm{wt} \%$.

\section{Surface modification of spirulina}

Deionized water $(44.80 \mathrm{~g})$ was added to spirulina powder $(5.00 \mathrm{~g})$ and dispersed in a beaker. Then, TTAB $(0.20 \mathrm{~g})$ was added into the spirulina dispersion and stirred for $30 \mathrm{~min}$. Then, the modified spirulina (T-spirulina), obtained after centrifugation, was added to deionized water to obtain a T-spirulina dispersion (100.00 g).

\section{Preparation of T-spirulina@PTFE/PSAN with a core-shell structure}

The PTFE/PSAN mixed emulsion (10.00 g), spirulina (5.00 g) and deionized water $(185 \mathrm{~g}$ ) were added and stirred for $30 \mathrm{~min}$, and the final product was designated as SP. The STP composite material was PTFE/PSAN microspheres adsorbed on the T-spirulina surface by a hetero-coagulation process. The PTFE/PSAN mixed emulsion $(10.00 \mathrm{~g})$, the previously prepared T-spirulina dispersion (100.00 g), $0.5 \mathrm{wt} \%$ formic acid (1.136 g) and deionized water were mixed and stirred for $30 \mathrm{~min}$. The total mass of the whole reaction system was $200.00 \mathrm{~g}$. The hetero-coagulation reaction process was completed at $98{ }^{\circ} \mathrm{C}$ with $600 \mathrm{rpm}$. The hetero-coagulation product was precipitated at the bottom of the water dispersion, and freeze-dried to remove the moisture. The final product was named STP10. The core-shell materials made using $20 \mathrm{~g}, 30 \mathrm{~g}, 40 \mathrm{~g}$ or $50 \mathrm{~g}$ of PTFE/PSAN mixed emulsion were designated as STP20, STP30, STP40 and STP50, respectively.

\section{Preparation of the EP/STP composite materials}

The AB glue is a type of EP. The STP10 (3.00 g) was weighed and uniformly dispersed in $\mathrm{AB}$ glue (57.00 g), which was cured by stirring at room temperature. Finally, the resulting composite material was designated as EP/STP10. The same preparation STP20, STP30, STP40, STP50 were also used and composite materials were designated as EP/ STP20, EP/STP30, EP/STP40 and EP/STP50, respectively. At the same time, using the previous steps, spirulina and pure PTFE were also made into corresponding control samples. Calculation of PTFE/PSAN emulsion residual rate: after the coagulation, the coagulation product sinks to the bottom of the water dispersion, and the upper liquid contains a small amount of and PTFE/PSAN mixed latex. Due to the boiling point of sulfuric acid $\left(338^{\circ} \mathrm{C}\right)$, an ordinary oven cannot be used to remove the $\mathrm{H}_{2} \mathrm{SO}_{4}$ solution. The acid base titration method was used to remove the mass of $\mathrm{H}_{2} \mathrm{SO}_{4}$. The concentration of $\mathrm{NaOH}$ solution was $0.01 \mathrm{~mol} \mathrm{~L}^{-1}$, and 20 $\mathrm{mL}$ of the supernatant solution was used to calculate the
$\mathrm{H}_{2} \mathrm{SO}_{4}$ content in the supernatant. The formula of the emulsion residual rate is:

$$
\begin{aligned}
\text { Residual rate }= & \left(\frac{M 2-\frac{0.01 \mathrm{~mol} \mathrm{~L}^{-1} \times V 2}{2 \times 20 \mathrm{~mL}} \times V 1 \times 98}{M 1+M 2-\frac{0.01 \mathrm{~mol} \mathrm{~L}^{-1} \times V 2}{2 \times 20 \mathrm{~mL}} \times V 1 \times 98}\right) \\
& \times 100 \%
\end{aligned}
$$

where $M 1$ is the quantity of coagulation products after drying, $M 2$ is quantity of supernatant after drying, $V 1$ is the volume of supernatant and $V 2$ is the volume of the $\mathrm{NaOH}$ solution. After spirulina was used to adsorb the PTFE/PSAN mixed emulsion, the hetero-coagulation product coagulated in the bottom of the water dispersion, and there was a small amount of PTFE/PSAN mixed emulsion in the supernatant. The boiling point of formic acid is $100.7{ }^{\circ} \mathrm{C}$, which can completely remove the formic acid solution. The formula for calculating the residual rate of the emulsion is:

$$
\text { Residual rate }=\frac{M 4}{M 3+M 4-5 g} \times 100 \%
$$

where $M 3$ is the quantity of hetero-coagulation products after drying and $M 4$ is the quantity of supernatant after drying.

\section{Characterization}

The chemical structures of PTFE/PSAN and PTFE samples were determined at room temperature using a UV-vis-NIR spectrophotometer (Cary 5000, Agilent). The thermal stability was determined under a nitrogen atmosphere using TGA (STA449 F3C, Netzsch). The samples were heated from ambient temperature to $800{ }^{\circ} \mathrm{C}$ at a heating rate of $10{ }^{\circ} \mathrm{C} \mathrm{min}^{-1}$. The microstructure of the sample was determined using a high resolution cold field emission scanning electron microscopy (Regulus8100, Hitachi). The zeta potentials of the heterocoagulation of the aqueous dispersions over time were measured using a Zetasizer nanosystem (90Plus, Brookhaven). The combustion behaviors of the materials and the controls were tested using a cone calorimeter (ZY6243, Zonsky Instruments). Specimens with a size of $100 \times 100 \times 3 \mathrm{~mm}^{3}$ were tested a heat flux of under $50 \mathrm{~kW} \mathrm{~m}^{-2}$, and the heat and smoke release information were recorded. The LOI value was measured on an oxygen index meter (JF-3, Nanjing Jiangning Analysis Instruments) according to a standard method: ISO 4589-2:1996. The specimens were prepared with sheet dimensions of $120 \times 6.5 \times$ $3 \mathrm{~mm}^{3}$. The vertical burning test was conducted on an instrument with sheet dimensions of $120 \times 13 \times 3 \mathrm{~mm}^{3}$ (CZF-3, Nanjing Jiangning Analysis Instrument). The tensile strength of the composite materials was measured at room temperature according to a standard method: ASTM D638-2014 using a universal testing machine (WDW-10G, Jinan Hengjin Testing Machines). The chemical oxygen demand (COD) of the upper residual liquid samples were calculated using spectrophotometry (DR5000, Hach). 


\section{Results and discussion}

In order to verify the feasibility of the subsequent heterocoagulation method effectively capturing highly dispersed microspheres, a simulation of using the preparation process of PTFE/PSAN in industry was created. The PTFE microspheres, which had stable chemical properties, were chosen as the target for removal, and surface modification with a sea urchin-like structure was simultaneously carried out, which gave an excellent dispersion performance in an aqueous emulsion. Fig. 1a is a schematic of the synthesis process for the PTFE/PSAN microspheres. The PSAN as a copolymer of acrylonitrile and styrene was randomly adhered on the PTFE surface, then emulsified by SDS and S13. This means that the sea urchin-like PTFE/PSAN microspheres can be highly dispersed in an aqueous solution. This proves that this hetero-coagulation process is suitable for the removal of microspheres in most hydrological environments as long as the highly dispersed PTFE/PSAN microspheres can be cleaned thoroughly afterwards. After dripping preemulsion, the mixed emulsion is heated to $75{ }^{\circ} \mathrm{C}$. The remaining initiator is added and the reaction is continued for $2-4 \mathrm{~h}$ to obtain the modified PTFE/PSAN emulsion.

It was important to confirm whether the PSAN copolymer effectively coats the PTFE particles, and so FTIR and X-ray
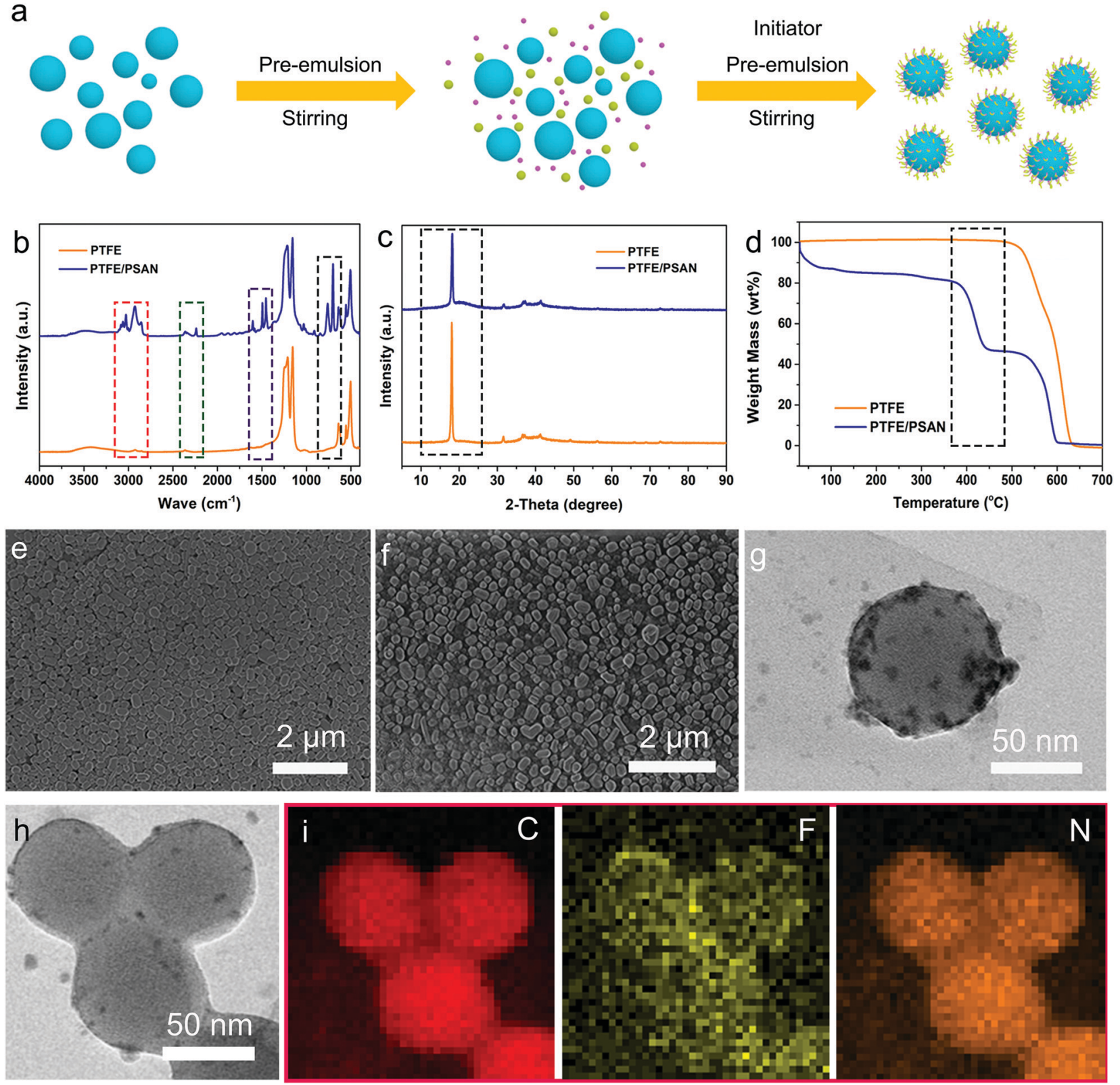

Fig. 1 (a) A schematic illustration of the process of synthesis of the PTFE/PSAN microspheres. (b) The FTIR spectra of the PTFE/PSAN and PTFE microspheres. (c) The XRD patterns of the PTFE/PSAN and PTFE specimens. (d) The TGA curves of the PTFE/PSAN and PTFE specimens. (e and f) The SEM images of PTFE and PTFE/PSAN microspheres. ( $g$ and $h$ ) The TEM images of PTFE/PSAN, and (i) EDS mapping images of the PTFE/PSAN microspheres. 
diffraction (XRD) were carried out to explore the structure of the PTFE/PSAN and neat PTFE samples. The FTIR spectra of these samples are shown in Fig. 1b. The stretching vibration peaks of PTFE and PTFE/PSAN containing C-F functional groups were mainly in the range of $1000-1300 \mathrm{~cm}^{-1}$. Comparing the PTFE/ PSAN with the PTFE, the main differences of the characteristic peaks were generated by functional groups represented as dashed boxes in Fig. 1b. The red dashed box indicates that the peak of PTFE/PSAN at about $2800-3200 \mathrm{~cm}^{-1}$ was caused by asymmetric stretching vibration of $-\mathrm{CH}_{3}$ in the PSAN copolymer. In the purple and black dashed boxes of Fig. 1b, the peaks at $1500-1600 \mathrm{~cm}^{-1}$ and $750-830 \mathrm{~cm}^{-1}$ represent the skeleton vibration of the benzene ring and $-\mathrm{CH}$ out-of-plane bending vibration, respectively. The PSAN copolymer also had a characteristic absorption peak of a cyano group $(-\mathrm{C} \equiv \mathrm{N})$ at $2245 \mathrm{~cm}^{-1}$.

Fig. 1c shows the XRD spectra of PTFE and PTFE/PSAN specimens. It was found that the PTFE had a sharp peak at $2 \theta=18^{\circ}$ because of its spiral structure, ${ }^{29}$ which was conducive to the efficient stacking of molecular chains forming crystals, rather than the planar zigzag shape of common polymers. The XRD curve of PTFE/PSAN also had sharp peaks at $2 \theta=18^{\circ}$, and there was an amorphous peak in the range of $2 \theta=19-27^{\circ}$, which indicated the existence of the PSAN copolymer as shown in the black dashed box in Fig. 1c.

From the TGA curve of PTFE sample in Fig. 1d, it can be concluded that the main weight loss of PTFE occurred between $550{ }^{\circ} \mathrm{C}$ and $650{ }^{\circ} \mathrm{C}$, which was mainly the decomposition and fracture of the $\mathrm{C}-\mathrm{C}$ and $\mathrm{C}-\mathrm{F}$ bonds. There were three main weight losses for the PTFE/PSAN sample. The first weight loss occurred mainly between $30{ }^{\circ} \mathrm{C}$ and $120{ }^{\circ} \mathrm{C}$, and the main weight loss was the volatilization of water molecules. The second weight loss was in the range of $360-450{ }^{\circ} \mathrm{C}$. The mass loss at this stage was mainly caused by the decomposition of the $\mathrm{C}-\mathrm{N}$ ring structure and the formation of char. The $\mathrm{C}-\mathrm{N}$ ring structure was dehydrogenated to carbon to form a stable polycyclic structure. ${ }^{30-32}$ It could be observed that between $450{ }^{\circ} \mathrm{C}$ and $605{ }^{\circ} \mathrm{C}$ that the PTFE part of PTFE/PSAN was about $47 \mathrm{wt} \%$ of the mass fraction. Fig. 1e and $\mathrm{f}$ are the SEM images of the PTFE and PTFE/PSAN microspheres, respectively. The PTFE microspheres were spherical or elliptical with a diameter between 100 and $250 \mathrm{~nm}$. Fig. $1 \mathrm{~g}$ is the TEM image of PTFE/ PSAN with sea urchin-like structure. It is observed that PSAN is outside of PTFE. The morphology of the PTFE/PSAN microspheres was basically similar to that of the PTFE microspheres. The energy dispersive spectroscopy (EDS) patterns of PTFE and PTFE/PSAN show that there are two elements of $\mathrm{C}$ and $\mathrm{F}$ in PTFE and the element masses of $\mathrm{C}$ and $\mathrm{F}$ were $45.30 \mathrm{wt} \%$, and $54.70 \mathrm{wt} \%$, respectively. But PTFE/PSAN has three elements of $\mathrm{C}, \mathrm{F}$ and $\mathrm{N}$, and the element masses of $\mathrm{C}, \mathrm{F}$ and $\mathrm{N}$ were $78.82 \mathrm{wt} \%, 14.71 \mathrm{wt} \%$ and $6.45 \mathrm{wt} \%$ (Fig. S1 and Table S1 $(\mathrm{ESI} \dagger))$. The surface of PTFE contained PSAN particles and so the PTFE/PSAN microspheres are called sea urchin-like. From the TEM images and the EDS mappings of PTFE/PSAN microspheres in Fig. 1h and i, it can be clearly seen that the PSAN copolymer was modified on the surface of the PTFE microspheres, which further indicated that the sea urchinlike PTFE/PSAN emulsion was successfully prepared.

The prepared PTFE/PSAN microspheres were usually coated with nonionic and anionic emulsifiers, such as S13 and SDS. This implied that that PTFE/PSAN emulsion was stable and difficult to coagulate. Spirulina has a high draw ratio and a large specific surface area, which favours surface modification. Compared with the zeta potential of other surfactants, TTAB is not only a cationic surfactant of spirulina, but also plays a important role as a demulsifier for the hetero-coagulation system.

Here, a little formic acid worked as an electrolyte to weaken the electrical double-layer structure of the PTFE/PSAN microsphere, which assisted the T-spirulina in breaking the equilibrium system of the emulsion and accelerated the heterocoagulation process. In Fig. 2a, it can be seen from the schematic diagram that a large number of PTFE/PSAN microspheres were coagulated on the surface of T-spirulina by electrostatic attraction, forming hetero-coagulation products with a coreshell structure. The mechanism of hetero-coagulation was mainly based on a diffuse electrical double layer model. ${ }^{33}$ With the assistance of formic acid, the TTAB on the T-spirulina surface completely destroyed the electrical double-layer structure of the PTFE/PSAN microspheres, so that the PTFE/PSAN microspheres were adsorbed on the T-spirulina surface.

From the zeta potentials observed in Fig. 2b, the zeta potential values increased significantly after adding T-spirulina and formic acid. The zeta potential value was $-84.22 \mathrm{mV}$ for the original PTFE/ PSAN emulsion, whereas the zeta potential value of the PTFE/PSAN emulsion $(200 \mathrm{~g})$ treated with $0.5 \mathrm{wt} \%$ formic acid $(1 \mathrm{~g})$ increased to $-68.68 \mathrm{mV}$. At this point, the PTFE/PSAN emulsion was still stable without any tendency to demulsify. After adding 0.1 wt $\%$ of spirulina, the zeta potential of the PTFE/PSAN emulsion increased to $-48.55 \mathrm{mV}$. When $0.1 \mathrm{wt} \%$ of T-spirulina, instead of spirulina, was added into the PTFE/PSAN emulsion, the emulsion had a tendency to demulsify and this zeta potential rapidly increased to $17.80 \mathrm{mV}$. This shows that TTAB plays a decisive role in promoting the coagulation of PTFE/PSAN microspheres on T-spirulina. Therefore, by adding $0.1 \mathrm{wt} \%$ of T-spirulina to $10 \mathrm{~g}$ of PTFE/PSAN emulsion, the zeta potential value is $29.26 \mathrm{mV}$. At this time, the PTFE/PSAN microspheres tended to demulsify in the solution, and were simultaneously adsorbed on the T-spirulina. Compared with the hetero-coagulation system of STP10, the zeta potentials of those in STP20, STP30, STP40, and STP50 decreased slightly, but they were all between $-30 \mathrm{mV}$ and $30 \mathrm{mV}$ (Fig. 2c). This was because of the increased content of PTFE/PSAN emulsion.

Fig. 2d shows the FTIR spectra of the upper residual liquid of the control, SP and STP10, STP20, STP30, STP40 and STP50. For the upper residual liquid of the control and SP after demulsification, there were $\mathrm{C}-\mathrm{F}$ absorption vibration peaks in the range of $1100-1300 \mathrm{~cm}^{-1}$ which indicated that the PTFE/ PSAN microspheres still remained in the upper residual liquid and that the hetero-coagulation process was incomplete.

Meanwhile, the upper residual liquid of STP10, STP20, STP30, STP40 and STP50 had no PTFE/PSAN microspheres remaining. Furthermore, the demulsification time of STP was 

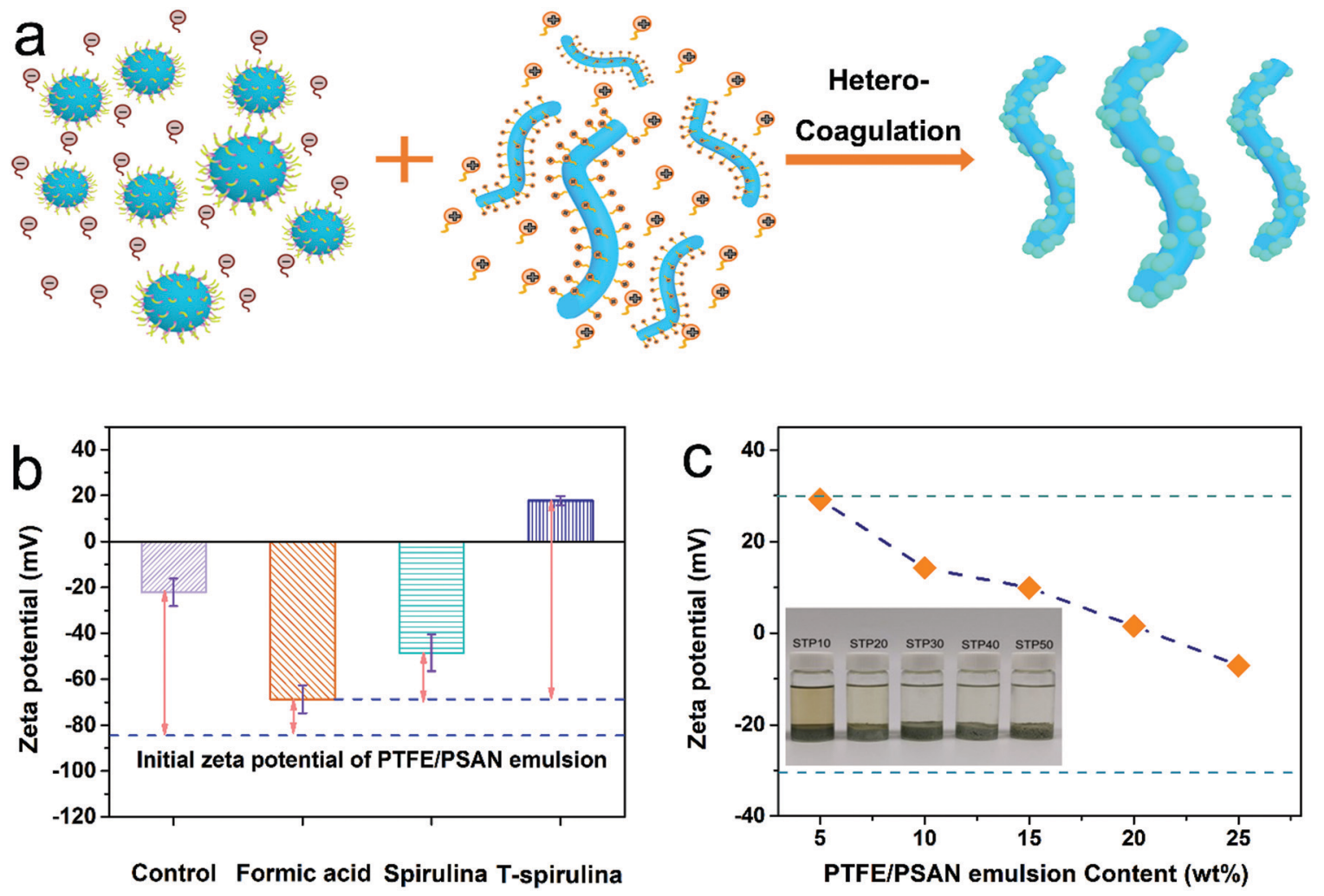

Control Formic acid Spirulina T-spirulina
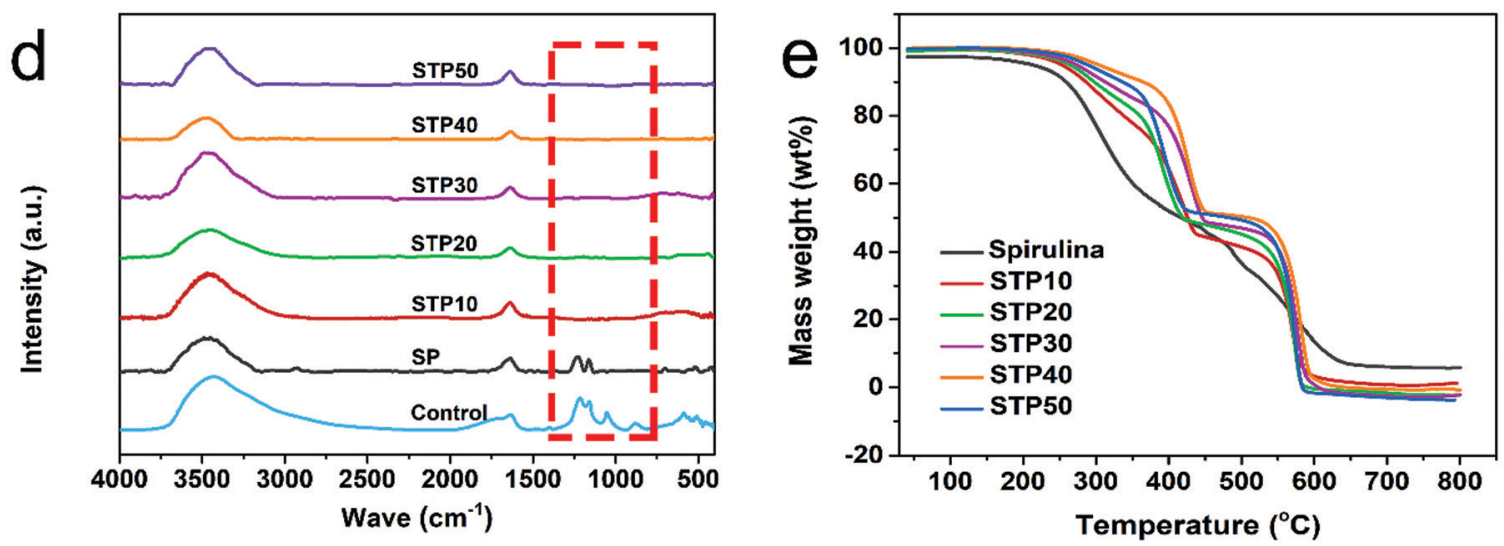

Fig. 2 (a) The schematic illustration of the fabrication of T-spirulina@PTFE/PSAN core-shell structure using a hetero-coagulation process. (b) The zeta potential curves of different hetero-coagulation dispersions. (c) The zeta potentials of core shells with different concentrations of PTFE/PSAN. The digital photographs show the termination status after the hetero-coagulation process. (d) The FTIR spectra of the upper liquid of the control, spirulina demulsification, STP10, STP20, STP30, STP40, and STP50. (e) TG curves of spirulina, STP10, STP20, STP30, STP40, STP50.

obviously faster than that of the control and SP samples (see Table S2 (ESI $\dagger)$ ). This effectively solved the problem of stubborn microplastics' removal, whereas the environmental pollution of the control sample caused by $\mathrm{H}_{2} \mathrm{SO}_{4}$ demulsification was avoided. The residual rate was calculated according to the formula shown previously, and it was found that after heterocoagulation of T-spirulina, the residual rate of PTFE/PSAN was almost $99.81 \mathrm{wt} \%$ (see Fig. S3 (ESI $\dagger$ )), whereas the residual rates of other demulsifiers such as $\mathrm{H}_{2} \mathrm{SO}_{4}, \mathrm{NaOH}$ and their complexes of $\mathrm{H}_{2} \mathrm{SO}_{4}$ with other cations were $8.94 \mathrm{wt} \%, 7.14 \mathrm{wt} \%$, $7.08 \mathrm{wt} \%, 3.29 \mathrm{wt} \%$, and $1.79 \mathrm{wt} \%$. The upper residual liquids of the control, SP, STP10, STP20, STP30 and STP50 were tested to determine the amount of COD present, and the results are shown in Fig. S4 (ESI $\dagger$ ). The COD of the SP supernatant was $90,745 \mathrm{mg} \mathrm{L}^{-1}$, whereas the CODs of STP10, STP20, STP30 and STP50 were 1115, 1595, 1990, $1703 \mathrm{mg} \mathrm{L}^{-1}$, respectively. Spirulina had a good removal effect on the PTFE/PSAN emulsion after being emulsified by TTAB. This also shows that TTAB is not only a cationic surfactant for spirulina, but also plays a role as a demulsifier for the hetero-coagulation system. Compared with the traditional coagulation process, the COD of the control was $2086 \mathrm{mg} \mathrm{L}^{-1}$, and the COD of STP was significantly lower than that of the control, which indicated that T-Spirulina had a better removal effect on the PTFE/PSAN emulsion. 
Fig. 2e shows thermal weight loss of spirulina and STP with different PTFE/PSAN loadings. The pyrolysis process of spirulina can be divided into three individual stages: drying, devolatilization and carbonization. In the first stage from 100-265 ${ }^{\circ} \mathrm{C}$, the weight loss of spirulina (11.9 wt\%) was due to the evaporation of internal moisture and light volatile
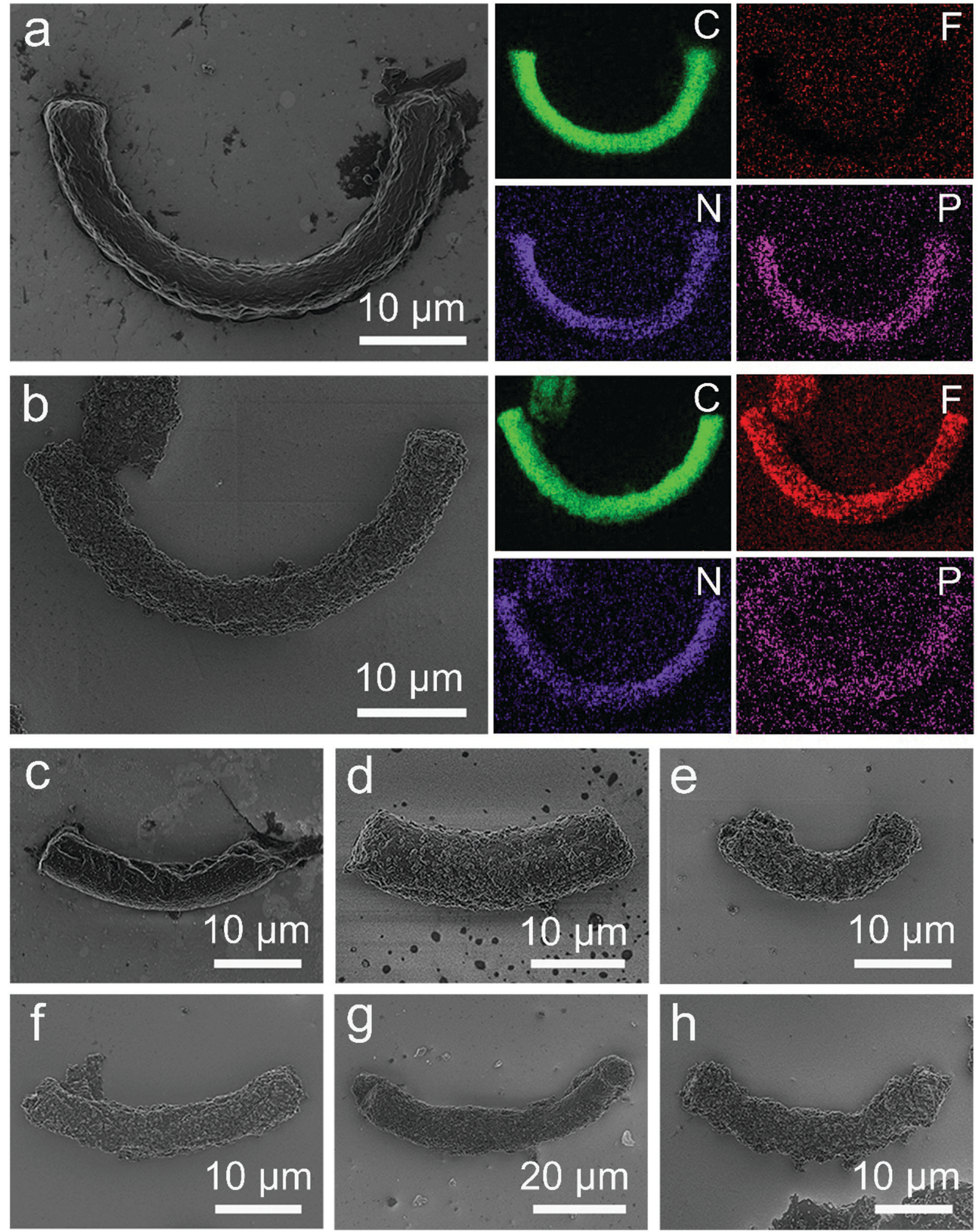

Fig. 3 (a) The SEM image and EDS mapping results for spirulina, (b) the SEM image and EDS mapping results for STP50, (c-h) the SEM images of SP, STP10, STP20, STP30, STP40 and STP50 samples. 
compounds. As the temperature increased, the polymer organic compounds further depolymerized and decomposed to form gas and coke. Thereafter, a significant weight loss (42.0 wt\%) occurred in the second stage at temperatures between 265$450{ }^{\circ} \mathrm{C}$ which was mainly derived from the thermal degradation of carbohydrates, proteins, and fats. ${ }^{34}$ When the temperature was above $450{ }^{\circ} \mathrm{C}$, the mass of spirulina still decreased by $35.0 \mathrm{wt} \%$. Non-volatile organic compounds may vaporize and form $\mathrm{CO}$ and $\mathrm{CO}_{2}$ due to the high temperature decarbonization. The final residue of spirulina was $5.71 \mathrm{wt} \%$ of non-volatile matter. The initial decomposition temperatures (95 wt\%) were 253.3, 264.6, 273.8, 304.4 and $288.9{ }^{\circ} \mathrm{C}$, corresponding to STP10, STP20, STP30, STP40 and STP50, respectively. The thermal decomposition had the same tendency. The main weight loss stages were $360.0-460.0{ }^{\circ} \mathrm{C}$ and 520.0-620 ${ }^{\circ} \mathrm{C}$, which were the thermal decomposition of PSAN and fracture of the $\mathrm{C}-\mathrm{C}$ and $\mathrm{C}-\mathrm{F}$ bonds in PTFE, respectively. From the thermal decomposition rates shown in Fig. S2 (ESI $\dagger$ ), it was also found that the thermal weight loss of the hetero-coagulation product samples were mainly at this stage. At about $750{ }^{\circ} \mathrm{C}$, the carbon residues of STP10, STP20, STP30, STP40, and STP50 were close to $0 \mathrm{wt} \%$. However, according to the weight loss of the $\mathrm{C}-\mathrm{C}$ and C-F bonds decomposition in PTFE at 520.0-620 ${ }^{\circ} \mathrm{C}$, the weight losses of STP10, STP20, STP30, STP40 and STP50 were 35.2, 42.3, 45.1, 50.0 and $51.1 \mathrm{wt} \%$, respectively, which indicated the increase of PTFE/PSAN contents adsorbed on the surface of the spirulina.

In order to study the effect of coating the PTFE/PSAN microspheres on the algae surface, hetero-coagulation products with added spirulina and T-spirulina were prepared. The results of the EDS characterization indicated that the spirulina alga and the microspheres had several elements (Fig. 3a and b), so that if the distribution of these elements are known, the distribution of microspheres on the samples will be known. The EDS mapping of the $\mathrm{C}, \mathrm{N}, \mathrm{P}$ and $\mathrm{F}$ elements were chosen as the tracers (as shown in Fig. $3 \mathrm{a}$ and b) to characterize the distribution of the PTFE/PSAN microspheres.

Fig. $3 \mathrm{a}-\mathrm{h}$ are the SEM images of spirulina and its heterocoagulation products. The shape of spirulina is crooked and cylindrical with a diameter of about $4 \mu \mathrm{m}$ and a length of $40 \mu \mathrm{m}$. The EDS characterization shows that spirulina contains $\mathrm{C}, \mathrm{N}$ and $\mathrm{P}$ elements. Fig. 3b is a SEM image of T-spirulina@PTFE/PSAN with a core-shell structure. The EDS mapping of the STP50 surface showed that it was abundant in $\mathrm{C}, \mathrm{N}$ and $\mathrm{F}$ elements, and only lacking $\mathrm{P}$ element. This further implied that the PTFE/PSAN microspheres had completely coated the surface of the T-spirulina. Fig. $3 \mathrm{c}$ and Fig. S5 (ESI $\dagger$ ) show the SP hetero-coagulation product of untreated spirulina with PTFE/PSAN microspheres. There was only a small number of PTFE/PSAN microspheres on the surface of the spirulina, which demonstrated that the hetero-coagulation effect was not ideal. Fig. 3d-h and Fig. S6 (ESI $\dagger$ ) are SEM images of STP10, STP20, STP30, STP40 and STP50 produced by the heterocoagulation process of T-spirulina with PTFE/PSAN microspheres. This demonstrates that the PTFE/PSAN microspheres could accumulate in an orderly fashion of the surface of T-spirulina under certain conditions.

In Fig. 4a, the stress-strain curves show that the failure strength of pure epoxy resin was $35.24 \mathrm{MPa}$, but, the failure strength of the
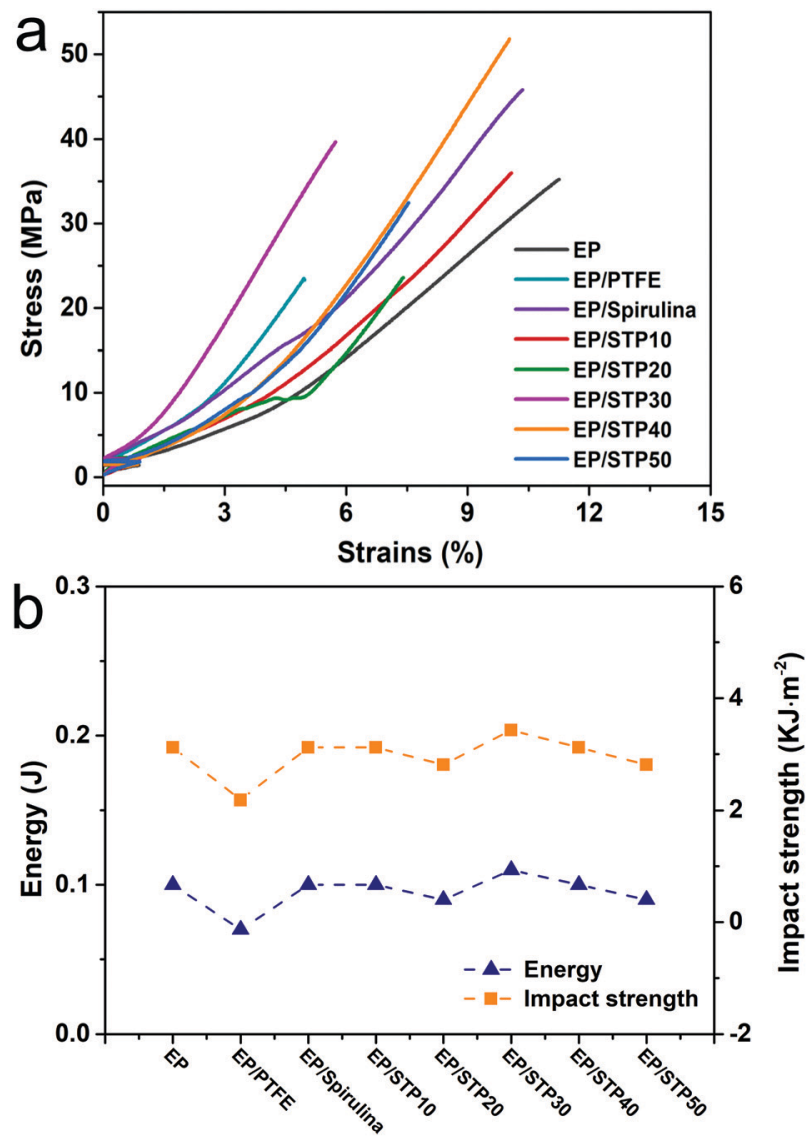

Fig. 4 (a) Tensile strength of different epoxy resin composite materials. (b) Impact strength of different epoxy resin composite materials.

EP/PTFE composite material decreased to $23.48 \mathrm{MPa}$ when $5 \mathrm{wt} \%$ of PTFE particles were added. Due to poor compatibility between the PTFE particles and the EP, this means that the PTFE was unable to disperse evenly in the EP. However, when $5 \mathrm{wt} \%$ of spirulina was added, the failure strength of the EP/spirulina composite material increased to $45.72 \mathrm{MPa}$, which indicated that spirulina was compatible for the base phase. When 5 wt $\%$ of STP10, STP20, STP30, STP40 and STP50 were added into the EP, the tensile curves showed that the failure strengths of their corresponding composites are 36.00, 24.13, 39.66, 51.62 and 32.47 MPa. When compared with the EP/PTFE composite material, spirulina and its derivative T-spirulina enhanced the mechanical strengths of the composite materials.

Fig. 4b shows the impact strength curves of different EP composite materials, which were consistent with the tensile strength results given previously. The impact strength of the EP/ PTFE composite material was $2.18 \mathrm{~kJ} \mathrm{~m}^{-2}$. The impact strengths of the EP, EP/Spirulina, EP/STP10, EP/STP20, EP/STP30, EP/STP40 and EP/STP50 samples were $3.12,3.12,3.12,2.81,3.43,3.12$ and $2.81 \mathrm{~kJ} \mathrm{~m}^{-2}$, respectively. Compared with that of the EP/PTFE sample, the impact resistances of the composite materials obviously had some advantage. After adding STP into the base phase, the impact of resistance of EP remained stable.

Fig. 5a shows the TGA curves of pure EP, EP/PTFE, EP/ Spirulina, EP/STP10, EP/STP20, EP/STP30, EP/STP40 and EP/ 

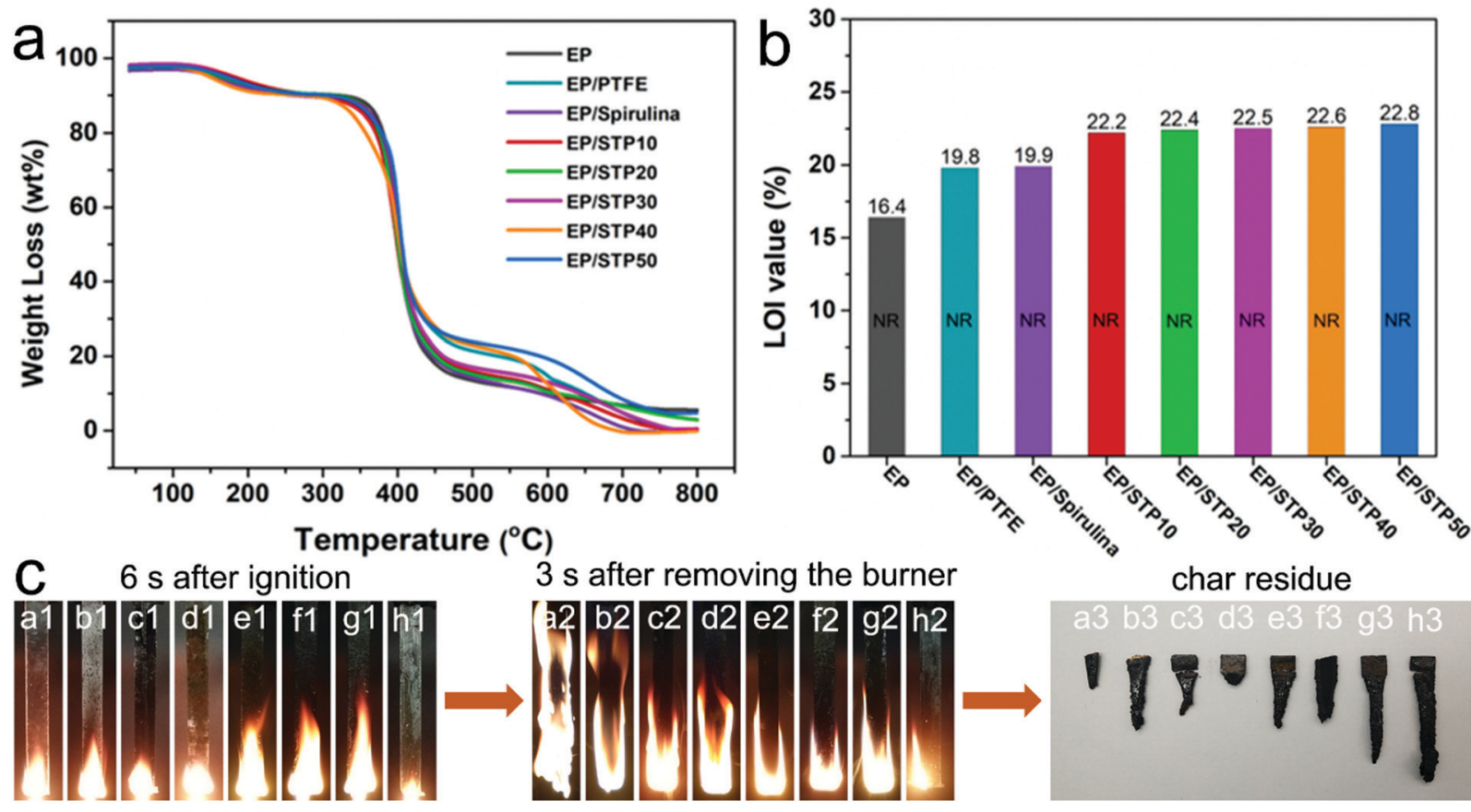

$3 \mathrm{~s}$ after removing the burner
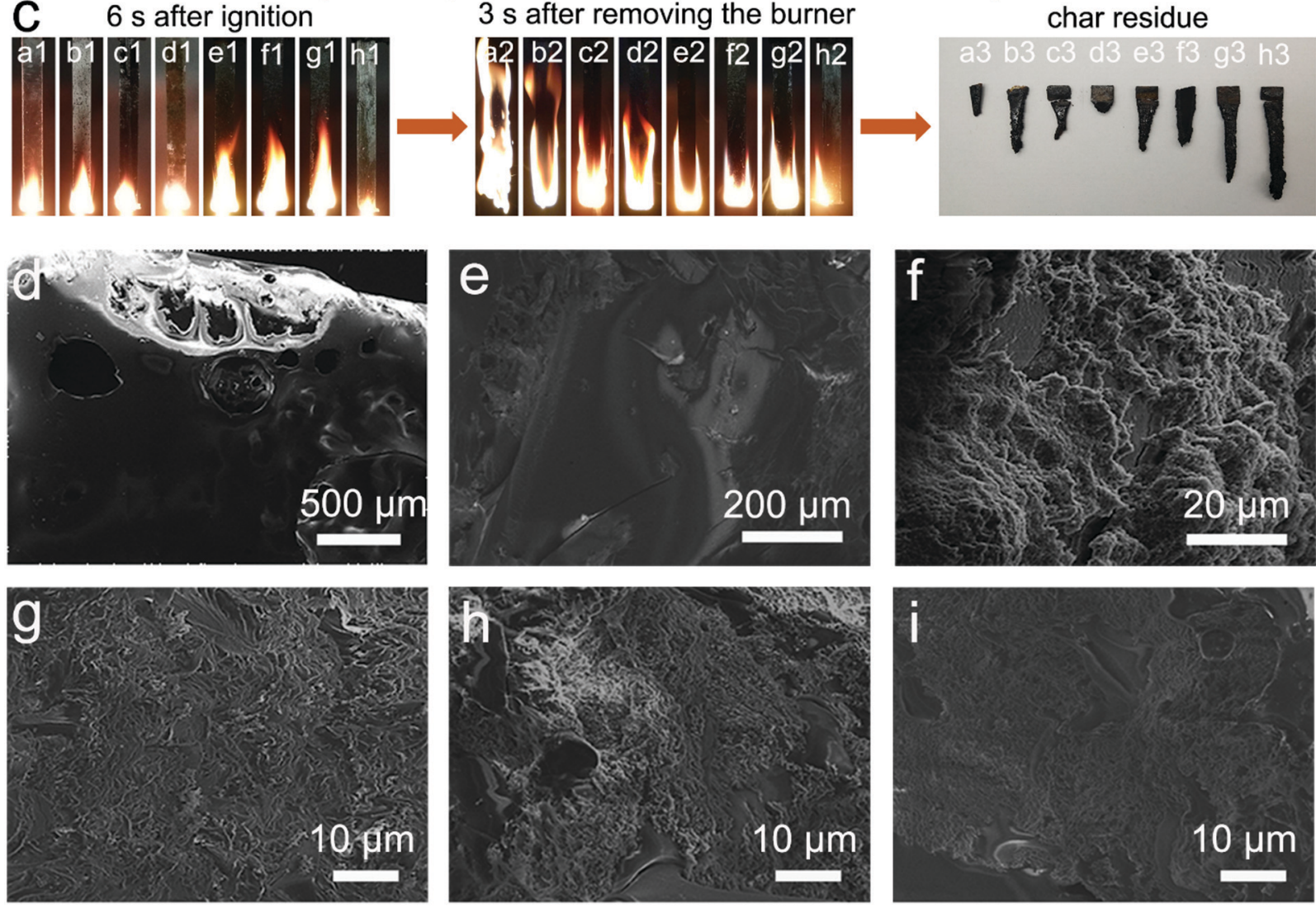

Fig. 5 (a) The TGA curves of EP, EP/PTFE, EP/Spirulina, EP/STP10, EP/STP20, EP/STP30, EP/STP40 and EP/STP50 samples. (b) The LOI results of EP, EP/ PTFE, EP/Spirulina, EP/STP10, EP/STP20, EP/STP30, EP/STP40, EP/STP50 samples. (c) The real time images of vertical flame tests for EP (a1-a3), EP/PTFE (b1-b3), EP/Spirulina (c1-c3), EP/STP10 (d1-d3), EP/STP20 (e1-e3), EP/STP30 (f1-f3), EP/STP40 (g1-g3) and EP/STP50 (h1-h3). (d-i) The SEM images of the residual char: EP (d), EP/Spirulina (e), EP/PTFE (f), EP/STP10 (g), EP/STP50 (h and i).

STP50 samples. Pure EP began to decompose at $366^{\circ} \mathrm{C}$, whereas all samples also showed their weight loss during $366-460{ }^{\circ} \mathrm{C}$. The pure EP sample lost nearly $75 \mathrm{wt} \%$ at this stage. Coke (5.68 wt\%) was produced at $800{ }^{\circ} \mathrm{C}$. In Fig. S7 (ESI $\dagger$ ), the same trend could also be found from the thermal decomposition rate loading of that same contents of PTFE, spirulina, STP10, STP20, STP30, STP40 and STP50, and the residual carbons at $550{ }^{\circ} \mathrm{C}$ were $20.00,11.80,13.60,13.40,15.44$ and $22.16 \mathrm{wt} \%$, which indicated that the addition of PTFE, spirulina and STP composite materials had a positive correlation for a flame retardant anti-dripping effect, especially with the loading of STP50. Admittedly, the final carbon contents of these composite samples were lower than that of pure EP at $800{ }^{\circ} \mathrm{C}$, and this is because of a combination of hydrogen radicals generated by combustion with small amounts of the $P$ element in spirulina, and the $F$ element in PTFE, which promoted the decomposition of the flame retardant materials. ${ }^{35}$ The combustion performance of EP and EP-based composite materials is shown in 
Fig. 5b. The LOI value of the pure EP sample was $16.4 \%$. For EP/ Spirulina and EP/PTFE composite materials, the LOI increased to $19.8 \%$ and $19.9 \%$, respectively, which was a significant increase. For a series of EP/STP composites, the LOI increased as the content of PTFE/PSAN in STP increased. Therefore, the LOI index value of the EP/STP50 composite material was up to $22.8 \%$. Fig. $5 \mathrm{c}$ shows real time images taken during the vertical burning test with EP (Fig. 5c(a1-a3)), EP/PTFE (Fig. 5c(b1-b3)), EP/Spirulina (Fig. 5c(c1-c3)), EP/STP10 (Fig. 5c(d1-d3)), EP/ STP20 (Fig. 5c(e1-e3)), EP/STP30 (Fig. 5c(f1-f3)), EP/STP40 (Fig. 5c(g1-g3)) and EP/STP50 (Fig. 5c(h1-h3)) samples.
In Fig. 5c(a1-a3), the pure EP was ignited quickly and burned strongly, and the flame spread rapidly even when the ignition source was removed. The whole combustion process lasted 80 s. After combustion, there was little residue left. It can be seen from Fig. 5c(b1-b3) that EP/PTFE (5 wt\%) was also ignited quickly and burned strongly while the flame spread rapidly after removing the source of the fire. However, after burning, there were many residues and the burning duration was $94 \mathrm{~s}$, but it was obvious that the PTFE added to the EP/PTFE sample delayed the flame spreading speed. Fig. 5(c1-c3) shows that EP/Spirulina sample was ignited at a slowe rate
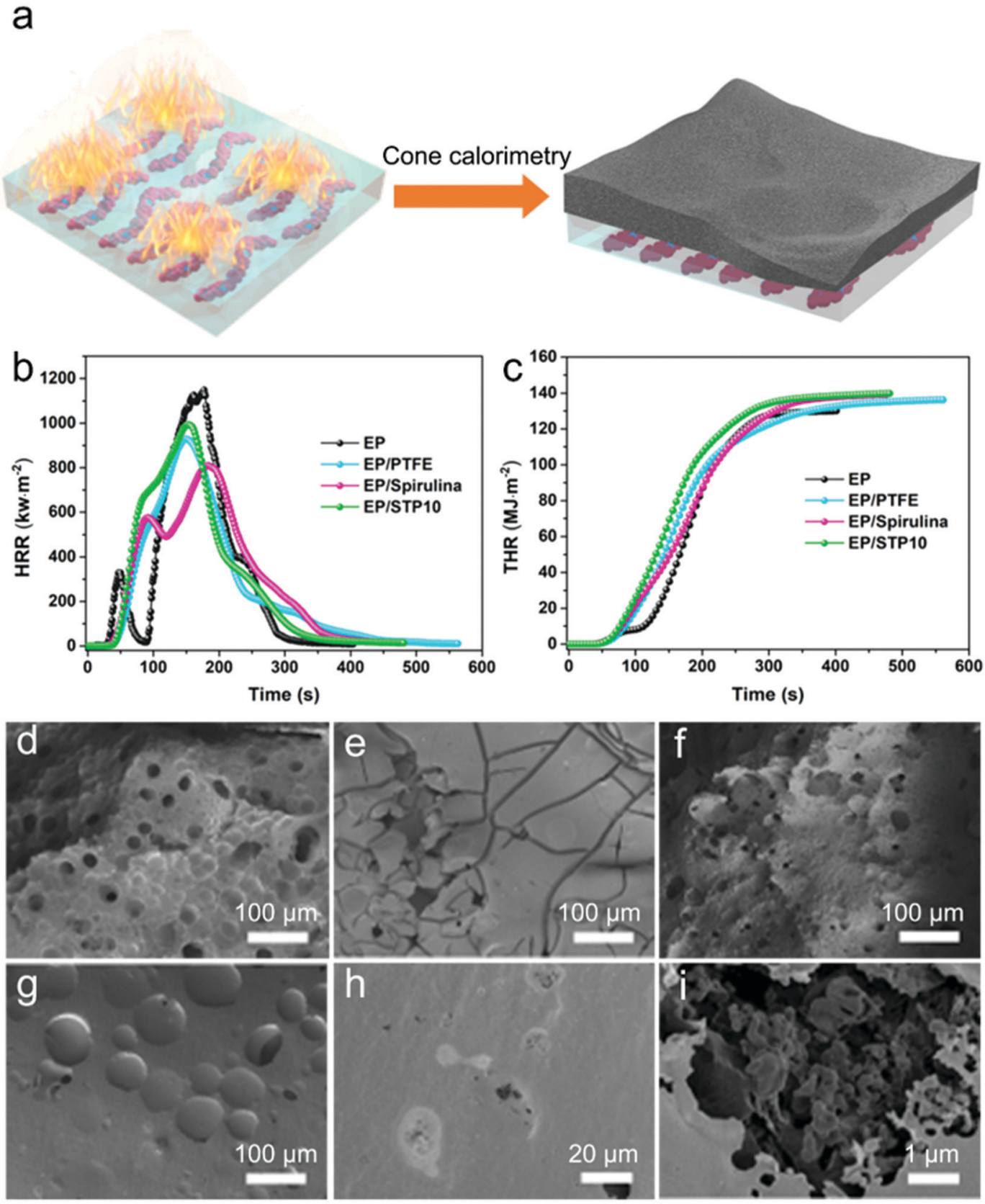

Fig. 6 (a) Schematic illustration of the cone calorimetry tests. (b) The HRRs and (c) THRs of EP, EP/PTFE, EP/Spirulina and EP/STP10 as a function of the burning time. The SEM images of residues after cone calorimetry tests: (d) EP, (e) EP/PTFE, (f) EP/Spirulina and (g-i) EP/STP10. 
and the combustion duration of th EP/Spirulina sample was about $109 \mathrm{~s}$. The EP/Spirulina sample was burned more gently than the pure EP sample, which indicated that spirulina has a good flame retardant performance. Although EP/STP20, EP/ STP30, EP/STP40 ignited faster, the flame spread was basically effective as the PTFE/PSAN coating, as the content increased and it was effectively a flame-retardant, which also showed that the PTFE/PSAN coating content has an effect on the flame retardant performance.

Combined with the SEM images of residual char, it can be seen from Fig. $5 \mathrm{~d}$ that there were holes with a diameter of about $400 \mu \mathrm{m}$ in the EP after combustion, which indicated that it was extremely combustible. The structure of EP/Spirulina after combustion is shown in Fig. 5e. This shows that no holes were formed on the surface of EP/Spirulina. This was due to the presence of extra nitrogen in the structure of Spirulina, which assisted with the decomposition of ammonia during the combustion process and improved the thermal oxidation stability. The resulting carbonaceous layer ${ }^{36}$ played a role in the heat insulation during the combustion process and also hindered the diffusion of oxygen, thereby slowing down pyrolysis at high temperatures. Fig. 5f shows the combustion products of EP/ PTFE. There were particles but no holes on the surface. Because of the fluoride produced by PTFE, the scavenging free radicals of the EP/PTFE have the ability to form a thin carbon layer to isolate oxygen and further achieve a flame retardant effect. Fig. 5g-i show the residual char of EP/STP10 and EP/STP50, respectively. It can be clearly seen that they are a layered structure and the presence of the PTFE/PSAN microsphere is also visible. This shows that the composite material added with a hetero-coagulation process not only has the function of scavenging free radicals, but also forms a dense carbon layer to preferentially isolate oxygen.

Fig. $6 \mathrm{a}$ is a schematic diagram of the cone calorimetry tests. The carbon layer structure has a good role in insulation and covers the surface of the polymer after the cone calorimetry tests. Fig. 6b shows the HRR of EP, EP/PSAN, EP/Spirulina and $\mathrm{EP} / \mathrm{STP} 10$. The neat EP sample has a distinct HRR curve and its peak of heat release rate (PHRR) reached up to $1108.7 \mathrm{~kW} \mathrm{~m}^{-2}$. In contrast, EP/Spirulina showed a wider HRR curve whereas its

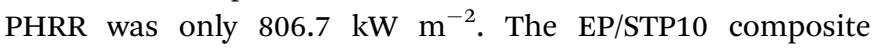
material had a wider HRR curve and its PHRR was 992.8 $\mathrm{kW} \mathrm{m} \mathrm{m}^{-2}$, which was slightly higher than the PHRR of the EP/PTFE composite material. This may be caused by the introduction of the PSAN copolymer, which promoted compatibility of the PTFE in the EP matrix. The EP/STP10 sample showed flame retardancy and anti-dripping performances. When the content of coated PTFE/PSAN increased, its PHRR also increased as shown in Fig. S9a (ESI $\dagger$ ).

The THR was used to measure the total heat release of materials during the combustion process, which was determined from combustible decomposition products. However, as shown in Fig. 6c and Fig. S9b (ESI $\dagger$ ), the total heat released by EP, EP/PTFE, EP/Spirulina, EP/STP10 and EP/STP50 was basically the same, which indicated that the EP matrix was completely burned. This indicated that the addition of PTFE,
Spirulina, STP10 and STP50 flame retardant materials cannot significantly reduce the total amount of decomposition.

At the same time, in order to study the toxicity of the combustion products, the production of $\mathrm{CO}$ was also obtained (Fig. S6a (ESI $\dagger$ )). Neat EP had a higher CO production, whereas that of the EP material added with spirulina was much lower. Fig. S6b (ESI $\dagger$ ) shows that the amount of $\mathrm{CO}_{2}$ produced in these samples depended on their burning time. The shape of the $\mathrm{CO}$ and $\mathrm{CO}_{2}$ curves were basically the same as the HRR curve, which indicated that most of the materials burned completely under the conditions of heat flow. Fig. S8 and S9 (ESI $\dagger$ ) show that the addition of PTFE, spirulina and STP could reduce the toxic combustion products, thereby reducing the risk of suffocation in a real fire. However, when STP50 was added to the EP matrix, the output of $\mathrm{CO}$ and $\mathrm{CO}_{2}$ was slightly increased, which was similar to that of pure EP. This was due to the content of PSAN copolymer following the increase of PTFE/PSAN content, thereby promoting the production of $\mathrm{CO}$ and $\mathrm{CO}_{2}$. Fig. $6 \mathrm{~d}$ shows an SEM image of the EP residues after the cone calorimetry tests. The surface of the EP residues after the cone calorimetry tests has a stable porous structure, which provided a channel for the propagation of oxygen starvation heat. The residues left after the cone calorimetry test of the sample of EP after adding PTFE were distributed in irregular blocks and rarely connected into one whole, but there were very few surface holes preventing the exchange of heat and oxygen. This also shows that PTFE is unevenly dispersed in the EP matrix.

Fig. 6f shows the residual carbon of EP/Spirulina after the cone calorimetry test, with only a few holes on this surface. From the EP/STP10 residues after cone calorimetry tests (Fig. 6g-i), the addition of STP10 can effectively improve the compatibility between PTFE and EP, and STP10 as the flame retardant hinders the burning of the EP matrix. Residual carbon of EP/STP50 after the cone calorimetry test (Fig. S10, $\mathrm{ESI} \dagger$ ) also shows similar trend. Some of the STP remain in the composite, which further illustrates that PTFE/PSAN has a flame retardant effect, even though it could not significantly reduce the total amount of EP decomposition.

\section{Conclusions}

This study proves that T-spirulina plays a decisive role in the preparation of STP during the hetero-coagulation process. With the assistance of formic acid, the modified spirulina absorbs at least three times its own mass of PTFE/PSAN microspheres during the hetero-coagulation process and, more importantly, the removal efficiency of the highly dispersed microspheres in water was up to $99.81 \mathrm{wt} \%$, which was better than other demulsifiers. The content of PTFE/PSAN was adjusted by the hetero-coagulation method to achieve the STP composite with a controllable thickness. This not only reduces the pollution of microplastics, but also turns waste into recyclables. Therefore, a series of STP composite materials were found to have flame retardant and anti-drip properties. The TGA tests results show that the addition of STP composites could improve the flame 
retardant performance of the EP matrix. Meanwhile, the LOI and vertical combustion tests show that the oxygen index value and vertical combustion performance also improve with the increase of the amount of PTFE/PSAN coating. With an addition of $5 \mathrm{wt} \%$ of STP50, the LOI value is 22.8 . Cone calorimetry tests showed that $\mathrm{HRR}, \mathrm{CO}$ and $\mathrm{CO}_{2}$ production were significantly decreased after PTFE, spirulina and STP10 were added, which was much lower than that of the neat EP materials. However, the HRR curve, $\mathrm{CO}$ and $\mathrm{CO}_{2}$ production of EP/STP50 was similar to that of pure EP materials. Due to the excessive coating of PTFE/PSAN, it barely reduces the combustion content, but forms a barrier to isolate oxygen and heat. This environmentally friendly and efficient treatment method not only contributes to environmental protection, but also provides a feasible solution for the treatment of microplastics for industry, and opens up prospects for applications involving fireproof and thermal insulation materials.

\section{Author contributions}

Shiyu Zhang conceived the idea and discussed it with Gaoda Zheng, Zhihao Cai, Ziyi Yu, Yuqin Yang, Hui Ye, Zheng Qin and Xiaobing Zuo; Gaoda Zheng performed the material synthesis, sample preparation and characterizations, and analyzed the data; Zhihao Cai performed the SEM measurements and analyzed the data; Gaoda Zheng, Ziyi Yu, Yuqin Yang, Hui Ye and Zheng Qin conducted in operando measurements and analyzed the data; Gaoda Zheng performed the cone calorimetry tests and analyzed the cone calorimetry data; Gaoda Zheng and Shiyu Zhang carried out the TEM and analyzed the data; Ziyi $\mathrm{Yu}$, and Yuqin Yang performed the zeta potential measurements and analyzed the data; Hui Ye and Zheng Qin performed the tensile strength measurements and analyzed the data; Gaoda Zheng and Shiyu Zhang wrote the preliminary draft of the manuscript with input from Zhihao Cai, Ziyi Yu, Yuqin Yang, Hui Ye and Zheng Qin, and they all discussed the results and revised the manuscript; All the authors contributed to the interpretation of the findings, and reviewing and commenting on the manuscript.

\section{Conflicts of interest}

The authors declare no competing financial interests.

\section{Acknowledgements}

This research is supported by general research project of basic science (natural science) in colleges and universities of Jiangsu Province (21KJB430033). This research is supported by Changshu Zhenfu New Materials Co., Ltd (China).

\section{References}

1 G. X. Wang, D. Huang, J. H. Ji, C. Völker and F. R. Wurm, Adv. Sci., 2020, 8.
2 D. S. Green, B. Boots, N. E. O'Connor and R. Thompson, Environ. Sci. Technol., 2017, 51, 68-77.

3 L. Mai, H. He, L. J. Bao, L. Y. Liu and E. Y. Zeng, Environ. Sci. Technol., 2020, 54, 15852-15860.

4 D. M. Mitrano and W. Wohlleben, Nat. Commun., 2020, 11, 5324 .

5 I. E. Napper and R. C. Thompson, Glob. Chall., 2020, 4, 1900081.

6 A. Kundu, N. P. Shetti, S. Basu, K. R. Reddy, M. N. Nadagouda and T. M. Aminabhavi, Chem. Eng. J., 2021, 421.

7 T. Narancic, S. Verstichel, S. Reddy Chaganti, L. MoralesGamez, S. T. Kenny, B. De Wilde, R. Babu Padamati and K. E. O’Connor, Environ. Sci. Technol., 2018, 52, 10441-10452.

8 S. Raikova, T. D. J. Knowles, M. J. Allen and C. J. Chuck, ACS Sustainable Chem. Eng., 2019, 7, 6769-6781.

9 R. A. Sheldon and M. Norton, Green Chem., 2020, 22, 6310-6322.

10 N. Tiwari, D. Santhiya and J. G. Sharma, Environ. Pollut., 2020, 265, 115044.

11 I. E. Napper, A. Bakir, S. J. Rowland and R. C. Thompson, Mar. Pollut. Bull., 2015, 99, 178-185.

12 A. Löhr, H. Savelli, R. Beunen, M. Kalz, A. Ragas and F. Van Belleghem, Curr. Opin. Environ. Sustain., 2017, 28, 90-99.

13 B. Liu, W. Zhao, Y. Shen, Y. Fan and Y. Wang, Langmuir, 2021, 37, 5993-6001.

14 W. Perren, A. Wojtasik and Q. Cai, ACS Omega, 2018, 3, 3357-3364.

15 G. Zhou, Q. Wang, J. Li, Q. Li, H. Xu, Q. Ye, Y. Wang, S. Shu and J. Zhang, Sci. Total Environ., 2021, 752, 141837.

16 C. Koti Reddy and D. Shailaja, Polym. Eng. Sci., 2015, 55, 499-505.

17 F. Mirza Esmaeili, M. S. Mortazavi and A. R. Dehghan Banadaki, Environ. Monit. Assess., 2019, 192, 42.

18 H. Chang, R. Hu, Y. Zou, X. Quan, N. Zhong, S. Zhao and Y. Sun, Water Res., 2020, 174.

19 A. L. Gonçalves, J. C. M. Pires and M. Simões, Algal Res., 2017, 24, 403-415.

20 S. Grosshagauer, K. Kraemer and V. Somoza, J. Agric. Food Chem., 2020, 68, 4109-4115.

21 S. Jabeen, X. Gao, M. Altarawneh, J.-I. Hayashi, M. Zhang and B. Z. Dlugogorski, Energy Fuels, 2019, 34, 474-482.

22 Y.-I. Park, M. Labrecque and J.-M. Lavoie, ACS Sustainable Chem. Eng., 2013, 1, 1348-1356.

23 J. Doke, V. Kalyan Raman and V. Ghole, Int. J. Algae, 2004, 6, 383-389.

24 A. Przystas, M. Jovic, K. A. Salmeia, D. Rentsch, L. Ferry, H. Mispreuve, H. Perler and S. Gaan, Polymers, 2018, 10.

25 Y. Zhou, W. He, N. Wang, D. Xu, X. Chen, M. He and J. Guo, Polym. Eng. Sci., 2019, 59, 1593-1602.

26 C. Hanumant Rao, A. Kothuru, A. P. Singh, B. K. S. V. L. Varaprasad and S. Goel, IEEE Trans. Compon., Packag., Manuf. Technol., 2020, 10, 1921-1928.

27 J. K. C. Valerio, H. Nakajima and M. R. Vasquez, Jpn. J. Appl. Phys., 2019, 58. 
28 C. Xie and K. Wang, Friction, 2020, 9, 1474-1491.

29 E. N. Brown and D. M. Dattelbaum, Polymer, 2005, 46, 3056-3068.

30 J. Guo, S.-Y. Mu, Y.-Y. Zhang and S. Zhang, AATCC J. Res., 2015, 4(2), 14-19.

31 M. Yu, C. Wang, Y. Zhao, M. Zhang and W. Wang, J. Appl. Polym. Sci., 2010, 116(2), 1207-1212.

32 X. Zhou, L. Bai, X. Liu, Y. Ren, Z. Song and X. Yang, J. Macromol. Sci., Part A: Pure Appl. Chem., 2019, 56, 1097-1103.
33 A. Alizadeh and M. Wang, Electrophoresis, 2020, 41, 1067-1072.

34 M. Dai, Z. Yu, S. Fang and X. Ma, Energy Convers. Manage., 2019, 192, 1-10.

35 X. Wang, S. Zhou, W.-W. Guo, P.-L. Wang, W. Xing, L. Song and Y. Hu, ACS Sustainable Chem. Eng., 2017, 5, 3409-3416.

36 Q. Liu, S. Yang, J. Ren and S. Ling, ACS Mater. Lett., 2020, 2, 712-720. 